\title{
Effect of Boron and Zinc Application on Nutrient Uptake in Guava (Psidium guajava L.) cv. Pant Prabhat Leaves
}

\author{
Rajkumar $^{1 *}$, J.P. Tiwari ${ }^{2}$, Shant Lal' ${ }^{2}$, Mohit Kumar ${ }^{2}$, Anshuman Singh ${ }^{1}$ and \\ Ashwani Kumar ${ }^{1}$ \\ ${ }^{1}$ ICAR- Central Soil Salinity Research Institute, Karnal - 132001, Haryana, India \\ ${ }^{2}$ Department of Horticulture, GBPUA \& T, Pantnagar - 263145, Uttarakhand, India \\ *Corresponding author
}

\section{A B S T R A C T}

An experiment was conducted to assess the influence of boron and zinc application on the nutrient status of guava cv. Pant Prabhat under tarai conditions at the foothills of the Himalayas. Boron and zinc were applied as foliar spray at four different concentrations viz. $\mathrm{ZnSO}_{4} @ 0 \%$ (control), 0.50\%,0.75\% and 1.0\% and $\mathrm{H}_{3} \mathrm{BO}_{3} @ 0 \%$ (control), 0.50\%, $0.75 \%$ and $1.0 \%$, respectively alone and in combinations. During both the years in the month of October, leaf $\mathrm{N}$ content was affected significantly. The maximum leaf $\mathrm{N}$ contents

\begin{tabular}{|c|}
\hline Keywords \\
\hline $\begin{array}{l}\text { Zinc, } \\
\text { Significantly, } \\
\text { Concentrations } \\
\text { and } \mathrm{ZnSO}_{4} \text {. }\end{array}$ \\
\hline Article Info \\
\hline $\begin{array}{l}\text { Accepted: } \\
\text { 23 May } 2017 \\
\text { Available Online: } \\
\text { 10 June } 2017\end{array}$ \\
\hline
\end{tabular}
$(2.38 \%$ and $2.18 \%)$ were recorded with the application of $\mathrm{ZnSO}_{4}(1.0 \%)+\mathrm{H}_{3} \mathrm{BO}_{3}$ (1.0\%). However, zinc and boron spray had non-significant effect on leaf $\mathrm{P}$ and $\mathrm{K}$ content in the month of July and October, respectively during both the years. Leaf zinc content increased significantly after the foliar application of various concentrations of $\mathrm{ZnSO}_{4}$. Maximum leaf zinc content (161.08 and $161.95 \mathrm{ppm})$ was obtained with $\mathrm{ZnSO}_{4}(1.0 \%)$ and $\mathrm{ZnSO}_{4}(1.0 \%)+\mathrm{H}_{3} \mathrm{BO}_{3}(1 \%)$ spray, respectively in July (after spray). After rainy season crop, maximum leaf zinc content (143.19 and $144.43 \mathrm{ppm}$ ) was observed with $1.0 \%$ $\mathrm{ZnSO}_{4}$ spray. While, after winter season crop, various concentrations of zinc and boron had non-significant on zinc content. However, various concentrations of zinc positively influenced the leaf zinc content with higher concentrations of zinc and boron. However, after winter season crop, non-significant differences were recorded. Boron content increased significantly with the increase in boron concentration during both the years of investigation but the interaction was non-significant. Maximum boron content (116.85 and $118.35 \mathrm{ppm})$ was observed after $\mathrm{ZnSO}_{4}(0.75 \%)+\mathrm{H}_{3} \mathrm{BO}_{3}(1.0 \%)$ foliar spray. However, after harvesting of rainy season crop, boron content of leaves differed significantly with various levels of boron concentration. The maximum boron content $(107.32 \mathrm{ppm})$ was observed in $\mathrm{ZnSO}_{4}(1.0 \%)+\mathrm{H}_{3} \mathrm{BO}_{3}(1.0 \%)$. These findings indicated that $\mathrm{ZnSO}_{4}(1.0 \%)+$ $\mathrm{H}_{3} \mathrm{BO}_{3}(1.0 \%)$ significantly influenced the $\mathrm{N}, \mathrm{Zn}$ and $\mathrm{B}$ concentrations in the leaves of guava cv. Pant Prabhat. However, zinc and boron spray at various concentrations had nonsignificant effect on leaf $\mathrm{P}$ and $\mathrm{K}$ content.

\section{Introduction}

Guava is the hardiest fruit crops among tropical fruit trees and excels most other fruit crops in productivity and adaptability. Due to its nutritional value, it is aptly referred to as
'Apple of tropics'. Guava ranks fourth in area and fifth in production among the most important fruits grown in India (Sharma et al., 2007). Guava is an excellent source of 
ascorbic acid, dietary fibre, pectin and minerals. Guava fruit contains water (80$82 \%)$, protein $(0.71 \%)$, fat $(0.5 \%)$, carbohydrate (11-13\%) and acids (2.4\%). Among fruits, it ranks third in vitamin-C content after Barbados cherry and Aonla. Guava fruits are rich in dietary fibres and vitamin $\mathrm{C}$ and have moderate levels of folic acid. Multiple health benefits of guava due to the presence of compounds like lycopene, quercitin, vitamin $\mathrm{C}$ and various polyphenols improves the immune system and protects against common infectious diseases (Rajkumar, 2016). A careful management is required to produce a profitable crop which includes cultural practices and obviously the fertilization or nutrition of an orchard.

Among the various factors, which affect the production and productivity of guava, macronutrient as well as micronutrients assumes great significance. Management of nutrients in guava refers to maintenance of the soil fertility and plant nutrient supply to an optimum level for sustaining the desired fruit quality through optimization of benefits from all the possible sources in integrated manner. The guava performs well in all categories of soil, but requirement of manures and fertilizers is guided to a greater extent by the categorization of soil i.e. low, medium and high. Earlier studies have shown better fruit yield, quality and best nutritional estimation methods, all contributing to the understanding of nutrient needs of trees and to develop methods to satisfy these needs. Guava is reported to develop characteristic deficiency symptoms in absence of $\mathrm{N}, \mathrm{P}, \mathrm{K}, \mathrm{Ca}, \mathrm{Mg}$ and $\mathrm{S}$ among macro-nutrients. Deficiencies of $\mathrm{Zn}$, $\mathrm{B}, \mathrm{Mn}, \mathrm{Fe}, \mathrm{Cu}$, and Mo among micronutrients are also reported. Inadequacy of either of these nutrients at critical stage of fruit development, adversely affect the productivity and quality of produce. Micronutrients help in the uptake of major nutrients and play an active role in the plant metabolism process starting from cell wall development to respiration, photosynthesis, chlorophyll formation, enzyme activity hormone synthesis, nitrogen fixation and reduction (Das 2003). The beneficial effect of zinc application has been well documented (Chhonkar and Singh 1981) in guava. Zinc is closely involved in the metabolism of RNA and ribosomal content in plant cells which lead to stimulation of carbohydrates, proteins and the DNA formation. It is also, induces pollen tube growth resulted from its role on tryptophan synthesis as an auxin precursor biosynthesis (Hassan et al., 2010). Zinc deficiency is common in tarai region of Uttarakhand and symptoms of its deficiency have been observed in many crops i.e., paddy, wheat and soybean. Despite this, research on more qualitative and quantitative aspects of guava trees seems to be related with micronutrients taking place at only a small number of centres. Singh et al., (1983) reported that boric acid has good effect on physico-chemical composition of guava. The deficiency of boron, second to zinc deficiency, has imparted a greater significance to boron amendment. An adequate boron amendment ensures not only ample fruit set, but guarantees optimum fruit yield with excellent quality in terms of juice content, ratio between total soluble solids and acidity, and fruit peel colour (Srivastava and Singh 2005). Very little information is available on effect of zinc and boron under agro-climatic conditions of Uttarakhand and no work so far has been done under tarai conditions with the cultivar Pant Prabhat. Therefore, it has become imperative to find out influence of zinc and boron on leaf nutrient status of guava.

\section{Materials and Methods}

The investigation was carried out at Horticultural Research Centre, Patherchatta, Govind Ballabh Pant University of 
Agriculture and Technology, Pantnagar, Distt. Udham Singh Nagar (Uttarakhand), Pantnagar situated at the foothills of the Himalayas at $29^{\circ}$ North latitude and $79.3^{\circ}$ East latitude. The altitude of the place is 243.84 meters above the mean sea level. This belt is characterized by hot summers and cool winters. The experiment was conducted on seven-year-old trees of guava cv. Pant Prabhat planted at $7 \times 7$ $\mathrm{m}$ distance in square system and maintained under uniform cultural practices. The experiment was laid out in Randomized Block Design.

The orchard soil is sandy loam in texture, $\mathrm{pH}$ 7.6, available $\mathrm{N}(276 \mathrm{~kg} / \mathrm{ha})$, available $\mathrm{P}_{2} \mathrm{O}_{5}$ $(30.24 \mathrm{~kg} / \mathrm{ha})$, available $\mathrm{K}_{2} \mathrm{O}(136.92 \mathrm{~kg} / \mathrm{ha})$, available zinc $(0.71 \mathrm{ppm})$ and available boron $(0.37 \mathrm{ppm}), \quad$ respectively. Both the micronutrients i.e. (boron and zinc) were applied as foliar spray alone or in combinations at four different concentrations viz. zinc sulphate $\left(\mathrm{ZnSO}_{4}\right) @ 0 \%$ (control), $0.50 \%, 0.75 \%$ and $1.00 \%$ and boric acid $\left(\mathrm{H}_{3} \mathrm{BO}_{3}\right) @ 0 \%$ (control), 0.50\%, 0.75\% and $1.00 \%$, respectively. The trees were sprayed with the help of a foot sprayer, using 0.1 per cent 'Teepol' as surfactant. The treatments were given in mid-July (single spray) and leaf sampling was done on $15^{\text {th }}$ July (before spray), $30^{\text {th }}$ July (after spray), $15^{\text {th }}$ October (after rainy season crop) and first week of December during both the years. The stock solutions were prepared by dissolving the required amount of $\mathrm{ZnSO}_{4}$ (neutralized with hydrated lime) and $\mathrm{H}_{3} \mathrm{BO}_{3}$ in distilled water. Five month old leaves from fifth position from the base of shoot were sampled from all directions of the tree with a sample size of 20 leaves per plant (Bhargava and Chadha, 1993). Sampling was done in first week of June before fertilizer application and first week of December for estimation of N, P and $\mathrm{K}$ during both the years of investigation. Leaves were gently washed in running tap water and then rinsed in $0.1 \mathrm{~N}$ hydrochloric acid and distilled water immediately after leaf sampling. The adhering water was blotted out with filter paper. Fresh weight of individual sample was taken before they were kept in oven at $60^{\circ} \mathrm{C}$ for 36 to $40 \mathrm{hrs}$ to get constant dry weight. After drying, the samples were grind in electric grinder and sieved through 40 mesh sieve size.

\section{Estimation of Nitrogen}

Total nitrogen was estimated by the "MicroKjeldahal Distillation" method. Two hundred gram of grind material of leaves was taken in "micro-Kjeldahal tube" in which 10-15 ml of conc. $\mathrm{H}_{2} \mathrm{SO}_{4}$ was added. Further $1 \mathrm{~g}$ digestion accelerator, $1 \mathrm{~g}$ salicylic acid and $1 \mathrm{~g}$ sodium thiosulphite were also added. The tubes were kept in digestion unit for digestion. After digestion, the material was taken for distillation and after distillation, distillate ammonia-metaborate was titrated against $0.4 \mathrm{~N} \mathrm{H}_{2} \mathrm{SO}_{4}$ (AOAC 1970).

\section{Estimation of phosphorus and potassium}

One gram of grind material of leaves was digested with $15 \mathrm{ml}$ of tri-acid mixture containing concentrated $\mathrm{HNO}_{3}, \mathrm{H}_{2} \mathrm{SO}_{4}$ and $60 \% \mathrm{HClO}_{4}$ in ratio of $10: 1: 3$ by volume as described by Jackson (1973) in digestion chamber (under ventilated hood). After digestion, filtration was done to remove the silica precipitate and volume was made upto $100 \mathrm{ml}$.

\section{Phosphorus}

Phosphorus content of the leaf was determined by "Vanadomolybdophosphoric yellow colour method" as described by Jackson (1973). Five $\mathrm{ml}$ of tri-acid digest aliquot was taken in $25 \mathrm{ml}$ volumetric flask to which $2.5 \mathrm{ml}$ of Bartons reagent was added and made up the volume $(25 \mathrm{ml})$. The intensity of yellow phosphovanadomolybdic complex 
was measured at $430 \mathrm{~nm}$ (Bosch and Lomb Spectronic-20 colorimeter). The standard curve was prepared by using $\mathrm{KH}_{2} \mathrm{PO}_{4}$. The phosphorus content was expressed on per cent dry weight basis.

\section{Estimation of potassium}

Total potassium content in the leaf samples was determined with the help of Flame Photometer. The results were expressed on per cent dry weight basis.

\section{Estimation of zinc}

Zinc was determined by directly feeding triacid digest in Atomic absorption spectrophotometer. The results were expressed in ppm (Lindsay and Norvell 1978).

\section{Estimation of boron}

A part (1.2 g) of well ground homogenous leaf sample was placed in clean silica crucibles and kept for ashing in a muffle furnace at $500^{\circ} \mathrm{C}$ for 3 hours.

The crucibles and contents were cooled and 2 $\mathrm{ml}$ of $2 \mathrm{M} \mathrm{HCl}$ was added into the crucible. The residue was allowed to stand for 30 minutes. The volume was made upto $12 \mathrm{ml}$ with distilled water and the suspension was filtered into plastic vials and extract was kept for total boron analysis.

Boron was estimated by taking $1 \mathrm{ml}$ aliquot of blank, diluted boron standard or sample solution into a plastic tube (10 ml capacity) and $2 \mathrm{ml}$ of buffer solution was added to it and solution was mixed. Two $\mathrm{ml}$ of azomethine- $\mathrm{H}$ reagent was added to it and the colour of the sample solution was read on spectrophotometer as described by Jackson (1973).

\section{Statistical analysis}

The data were analyzed by applying ' $F$ ' test and critical difference at 5 per cent level was calculated to compare the mean values of treatments for all the characters (OPSTAT Software, CCS HAU, Hisar.

\section{Results and Discussion}

The present results elucidate the effect of zinc and boron on leaf nutrient status of guava cv. Pant Prabhat after foliar application of zinc and boron. The various concentrations of zinc and boron did not affect the leaf $\mathrm{N}$ content non in July month during both the years.

\section{Nitrogen $(\mathbf{N})$}

The data presented in Table 1 revealed that leaf $\mathrm{N}$ content increased with increase in zinc and boron concentrations. The maximum leaf $\mathrm{N}$ contents $(2.22 \%$ and $2.15 \%)$ were recorded in treatment $\mathrm{T}_{16}$, while, the minimum leaf $\mathrm{N}$ content $(1.81 \%$ and $1.24 \%)$ was found with $\mathrm{T}_{1}$ (control) in the month of July during both the years but the interaction was non-significant. However, in the month of October the leaf $\mathrm{N}$ content was affected significantly during both the years. The maximum leaf $\mathrm{N}$ contents $(2.38 \%$ and 2.18 $\%$ ) were recorded in the treatment $\mathrm{T}_{16}$ followed by $\mathrm{T}_{12}$ in the first year, whereas, in the second year it was at par with $\mathrm{T}_{12}$. Minimum leaf $\mathrm{N}$ content (1.82\% and $1.27 \%)$ was found with $\mathrm{T}_{1}$ (control). These results are in accordance with the earlier findings of Manchanda (1974) who reported that zinc spray slightly increased $\mathrm{N}$ and $\mathrm{P}$ content in sweet orange leaves. Sharma and Bhattacharya (1989) also found that foliar application of zinc sulphate and chelamin significantly increased leaf $\mathrm{N}$ and $\mathrm{K}$

content of guava. Similarly, Supriya and Bhattacharya (1995) found that leaf $\mathrm{N}$ content 
increased with increasing concentration of zinc application in Assam lemon, whereas leaf $\mathrm{P}$ and $\mathrm{K}$ content showed just reverse results.

\section{Phosphorus (P)}

The data presented in Table 2 clearly indicated that foliar spray of zinc and boron did not affect the leaf phosphorus content. In the month of July during both the years, the maximum leaf $\mathrm{P}$ content $(0.171 \%$ and 0.191 $\%)$ was obtained with treatment $\mathrm{T}_{4}$. In the month of October, maximum leaf $\mathrm{P}$ contents $(0.258 \%$ and $0.257 \%)$ respectively, were recorded in the treatment $\mathrm{T}_{4}$ during both the years. Prakash et al., (2006) reported that various levels of $\mathrm{N}$, zinc and boron had significant effect on the concentration of $\mathrm{N}, \mathrm{P}$, $\mathrm{K}, \mathrm{Zn}$ and $\mathrm{B}$ in leaf tissues.

\section{Potassium (K)}

Zinc and boron spray had non-significant effect on leaf $\mathrm{K}$ content. The maximum leaf $\mathrm{K}$ contents $(1.357 \%$ and $1.39 \%)$ were recorded in the treatment $\mathrm{T}_{16}$, while minimum leaf $\mathrm{K}$ contents $(1.257 \%$ and $1.270 \%)$ were observed with the treatment $\mathrm{T}_{1}$ (control) in the month of July during both the years, respectively (Table 3 ). In the month of October, maximum leaf $\mathrm{K}$ content (1.087\% and $1.097 \%$ ) was recorded with treatment $\mathrm{T}_{16}$ followed by $\mathrm{T}_{12}$, while minimum leaf $\mathrm{K}$ content $(0.982 \%$ and $0.987 \%)$ was found in $\mathrm{T}_{1}$ (control) during both the years respectively. Similarly, Nijjar and Brar (1977) did not found significant effect of zinc application by different methods on the leaf $\mathrm{N}, \mathrm{P}, \mathrm{K}$ contents in Kinnow mandarin.

\section{Zinc (Zn)}

Leaf zinc content increased significantly after the foliar application of various concentrations of zinc sulphate. On the other hand, with the application of boron at various concentrations, zinc content did not differ significantly during $1^{\text {st }}$ year at various stages of experiment (Table 4). It was also observed that the maximum leaf zinc content (161.08 ppm) was obtained with treatment $T_{13}$ which was at par with $\mathrm{T}_{15}, \mathrm{~T}_{14}$ and $\mathrm{T}_{16}$ in the month of July (after spray), while minimum leaf zinc content (45.64 ppm) was recorded in treatment $\mathrm{T}_{4}$. In the month of October (After rainy season crop), maximum leaf zinc content (143.19 ppm) was observed in treatment $\mathrm{T}_{13}$ which was at par with $\mathrm{T}_{15}, \mathrm{~T}_{16}$ and $\mathrm{T}_{14}$ while, minimum leaf zinc content (45.08 ppm) was recorded with the treatment $\mathrm{T}_{4}$. After winter season crop, various concentrations of zinc and boron had nonsignificant on zinc content. However, various concentrations of zinc positively influenced the leaf zinc content with higher concentrations of zinc and boron (Table 4). The maximum leaf zinc content was observed in treatment $\mathrm{T}_{6}(59.04 \mathrm{ppm})$ followed by $\mathrm{T}_{7}$, $\mathrm{T}_{16}$ and $\mathrm{T}_{15}$, while, minimum leaf zinc content was observed in the treatment $\mathrm{T}_{2}(45.02 \mathrm{ppm})$. Higher content of zinc in leaves was reported with the application of zinc as observed by several workers (Kanwar and Dhingra, 1962; Smith, 1967; Manchanda et al., 1971 and Nijjar and Brar, 1977). However, Embleton et al., (1965) observed that zinc sulphate sprays did not consistently increase the zinc content of leaves.

During $2^{\text {nd }}$ year, similar results were obtained (Table 5). After foliar application, the zinc content significantly increased and found maximum in treatment $\mathrm{T}_{16}(161.95 \mathrm{ppm})$ which was at par with $\mathrm{T}_{13}, \mathrm{~T}_{14}$ and $\mathrm{T}_{15}$, while, the minimum leaf zinc content (47.21 ppm) was observed in $\mathrm{T}_{1}$ (control). After rainy season crop, the maximum leaf zinc content (144.43 ppm) was observed in treatment $T_{13}$ which was at par with $\mathrm{T}_{15}, \mathrm{~T}_{16}$ and $\mathrm{T}_{14}$, while, minimum leaf zinc content (46.34 ppm) was observed in $\mathrm{T}_{1}$ (control) which differed significantly. After winter season crop, nonsignificant differences were recorded. 
Table.1 Effect of zinc sulphate and boric acid on nitrogen content (\%) of leaves

\begin{tabular}{|l|c|c|c|c|}
\hline \multirow{2}{*}{ Treatments } & \multicolumn{4}{|c|}{ Nitrogen content (\%) } \\
\cline { 2 - 5 } & \multicolumn{2}{|c|}{$1^{\text {st }}$ Year } & \multicolumn{2}{c|}{$2^{\text {nd }}$ Year } \\
\cline { 2 - 5 } July & October & July & October \\
\hline$\left(\mathrm{T}_{1}\right) 0 \% \mathrm{ZnSO}_{4}+0 \% \mathrm{H}_{3} \mathrm{BO}_{3}$ & $1.813(7.73)$ & $1.820(7.75)$ & $1.243(6.40)$ & $1.270(6.47)$ \\
$(\mathrm{Control})$ & & & & \\
\hline$\left(\mathrm{T}_{2}\right) 0 \% \mathrm{ZnSO}_{4}+0.5 \% \mathrm{H}_{3} \mathrm{BO}_{3}$ & $1.897(7.91)$ & $1.897(8.10)$ & $1.573(7.06)$ & $1.600(7.27)$ \\
\hline$\left(\mathrm{T}_{3}\right) \% \mathrm{ZnSO}_{4}+0.75 \% \mathrm{H}_{3} \mathrm{BO}_{3}$ & $2.053(8.23)$ & $2.053(8.25)$ & $1.861(7.73)$ & $1.978(8.08)$ \\
\hline$\left(\mathrm{T}_{4}\right) 0 \% \mathrm{ZnSO}_{4}+1 \% \mathrm{H}_{3} \mathrm{BO}_{3}$ & $2.137(8.38)$ & $2.137(8.43)$ & $2.059(8.19)$ & $2.074(8.27)$ \\
\hline$\left(\mathrm{T}_{5}\right) 0.5 \% \mathrm{ZnSO}_{4}+0 \% \mathrm{H}_{3} \mathrm{BO}_{3}$ & $1.840(7.78)$ & $1.840(7.82)$ & $1.367(6.68)$ & $1.387(6.72)$ \\
\hline$\left(\mathrm{T}_{6}\right) 0.5 \% \mathrm{ZnSO}_{4}+0.5 \% \mathrm{H}_{3} \mathrm{BO}_{3}$ & $1.950(8.02)$ & $1.950(8.12)$ & $1.610(7.29)$ & $1.767(7.64)$ \\
\hline$\left(\mathrm{T}_{7}\right) 0.5 \% \mathrm{ZnSO}_{4}+0.75 \% \mathrm{H}_{3} \mathrm{BO}_{3}$ & $2.073(8.28)$ & $2.073(8.29)$ & $1.992(8.11)$ & $1.996(8.12)$ \\
\hline$\left(\mathrm{T}_{8}\right) 0.5 \% \mathrm{ZnSO}_{4}+1 \% \mathrm{H}_{3} \mathrm{BO}_{3}$ & $2.150(8.43)$ & $2.150(8.46)$ & $2.103(8.33)$ & $2.116(8.36)$ \\
\hline$\left(\mathrm{T}_{9}\right) 0.75 \% \mathrm{ZnSO}_{4}+0 \% \mathrm{H}_{3} \mathrm{BO}_{3}$ & $1.837(7.78)$ & $1.837(7.83)$ & $1.420(6.73)$ & $1.443(6.90)$ \\
\hline$\left(\mathrm{T}_{10}\right) 0.75 \% \mathrm{ZnSO}_{4}+0.5 \% \mathrm{H}_{3} \mathrm{BO}_{3}$ & $1.947(8.01)$ & $1.947(8.08)$ & $1.607(7.26)$ & $1.843(7.79)$ \\
\hline$\left(\mathrm{T}_{11}\right) 0.75 \% \mathrm{ZnSO}_{4}+0.75 \% \mathrm{H}_{3} \mathrm{BO}_{3}$ & $2.103(8.34)$ & $2.103(8.38)$ & $1.998(8.12)$ & $2.014(8.16)$ \\
\hline$\left(\mathrm{T}_{12}\right) 0.75 \% \mathrm{ZnSO}_{4}+1 \% \mathrm{H}_{3} \mathrm{BO}_{3}$ & $2.177(8.40)$ & $2.177(8.52)$ & $2.135(8.40)$ & $2.173(8.47)$ \\
\hline$\left(\mathrm{T}_{13}\right) 1 \% \mathrm{ZnSO}_{4}+0 \% \mathrm{H}_{3} \mathrm{BO}_{3}$ & $1.893(7.91)$ & $1.894(7.92)$ & $1.540(7.12)$ & $1.550(7.11)$ \\
\hline$\left(\mathrm{T}_{14}\right) 1 \% \mathrm{ZnSO}_{4}+0.5 \% \mathrm{H}_{3} \mathrm{BO}_{3}$ & $2.013(8.15)$ & $2.013(8.20)$ & $1.844(7.79)$ & $1.851(7.81)$ \\
\hline$\left(\mathrm{T}_{15}\right) 1 \% \mathrm{ZnSO}_{4}+0.75 \% \mathrm{H}_{3} \mathrm{BO}_{3}$ & $2.123(8.38)$ & $2.123(8.40)$ & $2.025(8.17)$ & $2.027(8.17)$ \\
\hline$\left(\mathrm{T}_{16}\right) 1 \% \mathrm{ZnSO}_{4}+1 \% \mathrm{H}_{3} \mathrm{BO}_{3}$ & $2.217(8.48)$ & $2.217(8.87)$ & $2.145(8.41)$ & $2.176(8.47)$ \\
\hline Sem \pm & $\mathbf{0 . 1 4 2}$ & $\mathbf{0 . 0 0 2}$ & $\mathbf{0 . 2 1 7}$ & $\mathbf{0 . 1 1 2}$ \\
\hline $\mathbf{C D}$ at 5\% & $\mathbf{N S}$ & $\mathbf{0 . 0 0 7}$ & $\mathbf{N S}$ & $\mathbf{0 . 3 2 2}$ \\
\hline
\end{tabular}

Figures in parentheses indicate transformed values (Arc sin)

Table.2 Effect of zinc sulphate and boric acid on phosphorus content (\%) of leaves

\begin{tabular}{|l|c|c|c|c|}
\hline \multirow{2}{*}{ Treatments } & \multicolumn{4}{|c|}{ Phosphorus content (\%) } \\
\cline { 2 - 5 } & \multicolumn{2}{|c|}{$1^{\text {st }}$ Year } & \multicolumn{2}{c|}{$2^{\text {nd }}$ Year } \\
\cline { 2 - 5 } $\begin{array}{l}\left(\mathrm{T}_{1}\right) 0 \% \mathrm{ZnSO}_{4}+0 \% \mathrm{H}_{3} \mathrm{BO}_{3} \\
(\mathrm{Control})\end{array}$ & $0.160(2.26)$ & $0.252(2.88)$ & $0.169(2.35)$ & $0.250(2.86)$ \\
\hline$\left(\mathrm{T}_{2}\right) 0 \% \mathrm{ZnSO}_{4}+0.5 \% \mathrm{H}_{3} \mathrm{BO}_{3}$ & $0.167(2.33)$ & $0.255(2.89)$ & $0.184(2.44)$ & $0.256(2.90)$ \\
\hline$\left(\mathrm{T}_{3}\right) 0 \% \mathrm{ZnSO}_{4}+0.75 \% \mathrm{H}_{3} \mathrm{BO}_{3}$ & $0.169(2.33)$ & $0.257(2.90)$ & $0.187(2.46)$ & $0.257(2.90)$ \\
\hline$\left(\mathrm{T}_{4}\right) 0 \% \mathrm{ZnSO}_{4}+1 \% \mathrm{H}_{3} \mathrm{BO}_{3}$ & $0.171(2.33)$ & $0.258(2.91)$ & $0.191(2.49)$ & $0.257(2.91)$ \\
\hline$\left(\mathrm{T}_{5}\right) 0.5 \% \mathrm{ZnSO}_{4}+0 \% \mathrm{H}_{3} \mathrm{BO}_{3}$ & $0.160(2.26)$ & $0.234(2.77)$ & $0.166(2.33)$ & $0.238(2.79)$ \\
\hline$\left(\mathrm{T}_{6}\right) 0.5 \% \mathrm{ZnSO}_{4}+0.5 \% \mathrm{H}_{3} \mathrm{BO}_{3}$ & $0.167(2.32)$ & $0.239(2.80)$ & $0.181(2.42)$ & $0.253(2.88)$ \\
\hline$\left(\mathrm{T}_{7}\right) 0.5 \% \mathrm{ZnSO}_{4}+0.75 \% \mathrm{H}_{3} \mathrm{BO}_{3}$ & $0.169(2.33)$ & $0.250(2.87)$ & $0.182(2.42)$ & $0.254(2.89)$ \\
\hline$\left(\mathrm{T}_{8}\right) 0.5 \% \mathrm{ZnSO}_{4}+1 \% \mathrm{H}_{3} \mathrm{BO}_{3}$ & $0.170(2.34)$ & $0.253(2.88)$ & $0.183(2.43)$ & $0.255(2.89)$ \\
\hline$\left(\mathrm{T}_{9}\right) 0.75 \% \mathrm{ZnSO}_{4}+0 \% \mathrm{H}_{3} \mathrm{BO}_{3}$ & $0.160(2.26)$ & $0.233(2.77)$ & $0.165(2.32)$ & $0.233(2.76)$ \\
\hline$\left(\mathrm{T}_{10}\right) 0.75 \% \mathrm{ZnSO}_{4}+0.5 \% \mathrm{H}_{3} \mathrm{BO}_{3}$ & $0.164(2.29)$ & $0.242(2.82)$ & $0.173(2.38)$ & $0.246(2.83)$ \\
\hline$\left(\mathrm{T}_{11}\right) 0.75 \% \mathrm{ZnSO}_{4}+0.75 \% \mathrm{H}_{3} \mathrm{BO}_{3}$ & $0.165(2.30)$ & $0.245(2.84)$ & $0.176(2.40)$ & $0.248(2.84)$ \\
\hline$\left(\mathrm{T}_{12}\right) 0.75 \% \mathrm{ZnSO}_{4}+1 \% \mathrm{H}_{3} \mathrm{BO}_{3}$ & $0.167(2.32)$ & $0.249(2.86)$ & $0.179(2.41)$ & $0.252(2.87)$ \\
\hline$\left(\mathrm{T}_{13}\right) 1 \% \mathrm{ZnSO}_{4}+0 \% \mathrm{H}_{3} \mathrm{BO}_{3}$ & $0.160(2.25)$ & $0.232(2.76)$ & $0.158(2.27)$ & $0.232(2.73)$ \\
\hline$\left(\mathrm{T}_{14}\right) 1 \% \mathrm{ZnSO}_{4}+0.5 \% \mathrm{H}_{3} \mathrm{BO}_{3}$ & $0.161(2.28)$ & $0.248(2.86)$ & $0.170(2.36)$ & $0.235(2.75)$ \\
\hline$\left(\mathrm{T}_{15}\right) 1 \% \mathrm{ZnSO}_{4}+0.75 \% \mathrm{H}_{3} \mathrm{BO}_{3}$ & $0.163(2.29)$ & $0.242(2.82)$ & $0.170(2.36)$ & $0.242(2.80)$ \\
\hline$\left(\mathrm{T}_{16}\right) 1 \% \mathrm{ZnSO}_{4}+1 \% \mathrm{H}_{3} \mathrm{BO}_{3}$ & $0.164(2.30)$ & $0.246(2.84)$ & $0.173(2.38)$ & $0.244(2.83)$ \\
\hline Sem \pm & $\mathbf{0 . 0 1 8}$ & $\mathbf{0 . 0 0 8}$ & $\mathbf{0 . 0 2 2}$ & $\mathbf{0 . 0 2 2}$ \\
\hline CD at 5\% & $\mathbf{N S}$ & $\mathbf{N S}$ & $\mathbf{N S}$ & NS \\
\hline
\end{tabular}

Figures in parentheses indicate transformed values (Arc sin) 
Table.3 Effect of zinc sulphate and boric acid on potassium content (\%) of leaves

\begin{tabular}{|c|c|c|c|c|}
\hline \multirow{3}{*}{ Treatments } & \multicolumn{4}{|c|}{ Potassium content (\%) } \\
\hline & \multicolumn{2}{|c|}{$1^{\text {st }}$ Year } & \multicolumn{2}{|c|}{$2^{\text {nd }}$ Year } \\
\hline & July & October & July & October \\
\hline $\begin{array}{l}\left(\mathrm{T}_{1}\right) 0 \% \mathrm{ZnSO}_{4}+0 \% \mathrm{H}_{3} \mathrm{BO}_{3} \\
\text { (Control) }\end{array}$ & $1.257(6.44)$ & $0.982(5.69)$ & $1.270(6.47)$ & $0.987(5.70)$ \\
\hline$\left(\mathrm{T}_{2}\right) 0 \% \mathrm{ZnSO}_{4}+0.5 \% \mathrm{H}_{3} \mathrm{BO}_{3}$ & $1.289(6.52)$ & $1.020(5.79)$ & $1.297(6.54)$ & $1.053(5.88)$ \\
\hline$\left(\mathrm{T}_{3}\right) 0 \% \mathrm{ZnSO}_{4}+0.75 \% \mathrm{H}_{3} \mathrm{BO}_{3}$ & $1.307(6.56)$ & $1.043(5.86)$ & $1.327(6.61)$ & $1.057(5.90)$ \\
\hline$\left(\mathrm{T}_{4}\right) 0 \% \mathrm{ZnSO}_{4}+1 \% \mathrm{H}_{3} \mathrm{BO}_{3}$ & $1.337(6.64)$ & $1.066(5.92)$ & $1.370(6.72)$ & $1.067(5.93)$ \\
\hline$\left(\mathrm{T}_{5}\right) 0.5 \% \mathrm{ZnSO}_{4}+0 \% \mathrm{H}_{3} \mathrm{BO}_{3}$ & $1.266(6.46)$ & $0.990(5.71)$ & $1.268(6.46)$ & $1.007(5.76)$ \\
\hline$\left(\mathrm{T}_{6}\right) 0.5 \% \mathrm{ZnSO}_{4}+0.5 \% \mathrm{H}_{3} \mathrm{BO}_{3}$ & $1.303(6.55)$ & $1.033(5.83)$ & $1.313(6.58)$ & $1.047(5.87)$ \\
\hline$\left(\mathrm{T}_{7}\right) 0.5 \% \mathrm{ZnSO}_{4}+0.75 \% \mathrm{H}_{3} \mathrm{BO}_{3}$ & $1.321(6.60)$ & $1.050(5.88)$ & $1.330(6.62)$ & $1.057(5.90)$ \\
\hline$\left(\mathrm{T}_{8}\right) 0.5 \% \mathrm{ZnSO}_{4}+1 \% \mathrm{H}_{3} \mathrm{BO}_{3}$ & $1.338(6.64)$ & $1.070(5.94)$ & $1.367(6.71)$ & $1.073(5.95)$ \\
\hline$\left(\mathrm{T}_{9}\right) 0.75 \% \mathrm{ZnSO}_{4}+0 \% \mathrm{H}_{3} \mathrm{BO}_{3}$ & $1.263(6.45)$ & $1.010(5.77)$ & $1.273(6.48)$ & $1.013(5.78)$ \\
\hline$\left(\mathrm{T}_{10}\right) 0.75 \% \mathrm{ZnSO}_{4}+0.5 \% \mathrm{H}_{3} \mathrm{BO}_{3}$ & $1.296(6.53)$ & $1.034(5.84)$ & $1.303(6.55)$ & $1.053(5.88)$ \\
\hline$\left(\mathrm{T}_{11}\right) 0.75 \% \mathrm{ZnSO}_{4}+0.75 \% \mathrm{H}_{3} \mathrm{BO}_{3}$ & $1.324(6.61)$ & $1.059(5.91)$ & $1.333(6.63)$ & $1.061(5.91)$ \\
\hline$\left(\mathrm{T}_{12}\right) 0.75 \% \mathrm{ZnSO}_{4}+1 \% \mathrm{H}_{3} \mathrm{BO}_{3}$ & $1.341(6.65)$ & $1.077(5.95)$ & $1.383(6.75)$ & $1.083(5.97)$ \\
\hline$\left(\mathrm{T}_{13}\right) 1 \% \mathrm{ZnSO}_{4}+0 \% \mathrm{H}_{3} \mathrm{BO}_{3}$ & $1.274(6.48)$ & $1.010(5.77)$ & $1.280(6.49)$ & $1.043(5.86)$ \\
\hline$\left(\mathrm{T}_{14}\right) 1 \% \mathrm{ZnSO}_{4}+0.5 \% \mathrm{H}_{3} \mathrm{BO}_{3}$ & $1.306(6.56)$ & $1.037(5.84)$ & $1.313(6.58)$ & $1.053(5.89)$ \\
\hline$\left(\mathrm{T}_{15}\right) 1 \% \mathrm{ZnSO}_{4}+0.75 \% \mathrm{H}_{3} \mathrm{BO}_{3}$ & $1.322(6.60)$ & $1.063(5.91)$ & $1.347(6.66)$ & $1.060(5.91)$ \\
\hline$\left(\mathrm{T}_{16}\right) 1 \% \mathrm{ZnSO}_{4}+1 \% \mathrm{H}_{3} \mathrm{BO}_{3}$ & $1.357(6.69)$ & $1.087(5.98)$ & $1.390(6.77)$ & $1.097(6.01)$ \\
\hline Sem \pm & 0.038 & 0.027 & 0.046 & 0.042 \\
\hline CD at $5 \%$ & NS & NS & NS & NS \\
\hline
\end{tabular}

Figures in parentheses indicate transformed values (Arc sin)

Table.4 Effect of zinc sulphate and boric acid on zinc content (ppm) of leaves During first year at various stages of the experiment

\begin{tabular}{|c|c|c|c|c|}
\hline \multirow[b]{2}{*}{ Treatments } & \multicolumn{4}{|c|}{ Zinc content (ppm) during first year } \\
\hline & Before spray & After spray & $\begin{array}{l}\text { After rainy } \\
\text { season crop }\end{array}$ & $\begin{array}{l}\text { After winter } \\
\text { season crop }\end{array}$ \\
\hline $\begin{array}{l}\left(\mathrm{T}_{1}\right) 0 \% \% \quad \mathrm{ZnSO}_{4}+0 \% \mathrm{H}_{3} \mathrm{BO}_{3} \\
\text { (Control) }\end{array}$ & 45.21 & 45.91 & 45.52 & 45.86 \\
\hline$\left(\mathrm{T}_{2}\right) 0 \% \mathrm{ZnSO}_{4}+0.5 \% \mathrm{H}_{3} \mathrm{BO}_{3}$ & 45.50 & 48.49 & 47.12 & 45.03 \\
\hline$\left(\mathrm{T}_{3}\right) 0 \% \mathrm{ZnSO}_{4}+0.75 \% \mathrm{H}_{3} \mathrm{BO}_{3}$ & 45.91 & 46.26 & 45.40 & 45.35 \\
\hline$\left(\mathrm{T}_{4}\right) 0 \% \mathrm{ZnSO}_{4}+1 \% \mathrm{H}_{3} \mathrm{BO}_{3}$ & 45.17 & 45.64 & 45.08 & 45.42 \\
\hline$\left(\mathrm{T}_{5}\right) 0.5 \% \mathrm{ZnSO}_{4}+0 \% \mathrm{H}_{3} \mathrm{BO}_{3}$ & 45.04 & 72.66 & 72.34 & 50.88 \\
\hline$\left(\mathrm{T}_{6}\right) 0.5 \% \mathrm{ZnSO}_{4}+0.5 \% \mathrm{H}_{3} \mathrm{BO}_{3}$ & 47.00 & 87.89 & 72.29 & 59.04 \\
\hline$\left(\mathrm{T}_{7}\right) 0.5 \% \mathrm{ZnSO}_{4}+0.75 \% \mathrm{H}_{3} \mathrm{BO}_{3}$ & 46.34 & 86.26 & 72.60 & 54.12 \\
\hline$\left(\mathrm{T}_{8}\right) 0.5 \% \mathrm{ZnSO}_{4}+1 \% \mathrm{H}_{3} \mathrm{BO}_{3}$ & 46.99 & 80.08 & 72.16 & 47.93 \\
\hline$\left(\mathrm{T}_{9}\right) 0.75 \% \mathrm{ZnSO}_{4}+0 \% \mathrm{H}_{3} \mathrm{BO}_{3}$ & 47.06 & 129.74 & 114.17 & 53.28 \\
\hline$\left(\mathrm{T}_{10}\right) 0.75 \% \mathrm{ZnSO}_{4}+0.5 \% \mathrm{H}_{3} \mathrm{BO}_{3}$ & 46.96 & 130.52 & 114.03 & 52.83 \\
\hline$\left(\mathrm{T}_{11}\right) 0.75 \% \mathrm{ZnSO}_{4}+0.75 \% \mathrm{H}_{3} \mathrm{BO}_{3}$ & 45.15 & 131.95 & 115.40 & 52.56 \\
\hline$\left(\mathrm{T}_{12}\right) 0.75 \% \mathrm{ZnSO}_{4}+1 \% \mathrm{H}_{3} \mathrm{BO}_{3}$ & 47.75 & 132.06 & 115.74 & 52.35 \\
\hline$\left(\mathrm{T}_{13}\right) 1 \% \mathrm{ZnSO}_{4}+0 \% \mathrm{H}_{3} \mathrm{BO}_{3}$ & 47.20 & 161.08 & 143.19 & 49.88 \\
\hline$\left(\mathrm{T}_{14}\right) 1 \% \mathrm{ZnSO}_{4}+0.5 \% \mathrm{H}_{3} \mathrm{BO}_{3}$ & 46.68 & 157.89 & 141.85 & 52.00 \\
\hline$\left(\mathrm{T}_{15}\right) 1 \% \mathrm{ZnSO}_{4}+0.75 \% \mathrm{H}_{3} \mathrm{BO}_{3}$ & 45.64 & 158.04 & 142.53 & 53.26 \\
\hline$\left(\mathrm{T}_{16}\right) 1 \% \mathrm{ZnSO}_{4}+1 \% \mathrm{H}_{3} \mathrm{BO}_{3}$ & 46.91 & 157.57 & 142.43 & 53.64 \\
\hline Sem \pm & 1.175 & 6.302 & 4.264 & $\mathbf{3 . 5 8 0}$ \\
\hline CD at $5 \%$ & NS & 18.202 & 12.318 & NS \\
\hline
\end{tabular}

Figures in parentheses indicate transformed values (Arc sin) 
Table.5 Effect of zinc sulphate and boric acid on zinc content (ppm) of leaves During second year at various stages of the experiment

\begin{tabular}{|l|c|c|c|c|}
\hline \multirow{2}{*}{ Treatments } & \multicolumn{3}{|c|}{ Zinc content (ppm) during second year } \\
\cline { 2 - 5 } & Before spray & After spray & $\begin{array}{c}\text { After rainy } \\
\text { season crop }\end{array}$ & $\begin{array}{c}\text { After winter } \\
\text { season crop }\end{array}$ \\
\hline $\begin{array}{l}\left(\mathrm{T}_{1}\right) 0 \% \mathrm{ZnSO}_{4}+0 \% \mathrm{H}_{3} \mathrm{BO}_{3} \\
(\mathrm{Control})\end{array}$ & 46.25 & 47.21 & 46.34 & 45.05 \\
\hline$\left(\mathrm{T}_{2}\right) 0 \% \mathrm{ZnSO}_{4}+0.5 \% \mathrm{H}_{3} \mathrm{BO}_{3}$ & 45.81 & 50.43 & 46.99 & 45.70 \\
\hline$\left(\mathrm{T}_{3}\right) 0 \% \mathrm{ZnSO}_{4}+0.75 \% \mathrm{H}_{3} \mathrm{BO}_{3}$ & 47.61 & 51.11 & 50.64 & 47.96 \\
\hline$\left(\mathrm{T}_{4}\right) 0 \% \mathrm{ZnSO}_{4}+1 \% \mathrm{H}_{3} \mathrm{BO}_{3}$ & 45.18 & 50.61 & 52.04 & 51.76 \\
\hline$\left(\mathrm{T}_{5}\right) 0.5 \% \mathrm{ZnSO}_{4}+0 \% \mathrm{H}_{3} \mathrm{BO}_{3}$ & 46.78 & 88.84 & 77.30 & 53.01 \\
\hline$\left(\mathrm{T}_{6}\right) 0.5 \% \mathrm{ZnSO}_{4}+0.5 \% \mathrm{H}_{3} \mathrm{BO}_{3}$ & 45.95 & 88.96 & 72.45 & 52.40 \\
\hline$\left(\mathrm{T}_{7}\right) 0.5 \% \mathrm{ZnSO}_{4}+0.75 \% \mathrm{H}_{3} \mathrm{BO}_{3}$ & 44.92 & 87.25 & 75.82 & 52.06 \\
\hline$\left(\mathrm{T}_{8}\right) 0.5 \% \mathrm{ZnSO}_{4}+1 \% \mathrm{H}_{3} \mathrm{BO}_{3}$ & 45.91 & 82.50 & 75.64 & 53.21 \\
\hline$\left(\mathrm{T}_{9}\right) 0.75 \% \mathrm{ZnSO}_{4}+0 \% \mathrm{H}_{3} \mathrm{BO}_{3}$ & 47.60 & 130.34 & 115.45 & 48.36 \\
\hline$\left(\mathrm{T}_{10}\right) 0.75 \% \mathrm{ZnSO}_{4}+0.5 \% \mathrm{H}_{3} \mathrm{BO}_{3}$ & 46.59 & 138.11 & 115.64 & 48.97 \\
\hline$\left(\mathrm{T}_{11}\right) 0.75 \% \mathrm{ZnSO}_{4}+0.75 \% \mathrm{H}_{3} \mathrm{BO}_{3}$ & 46.13 & 131.41 & 116.33 & 53.50 \\
\hline$\left(\mathrm{T}_{12}\right) 0.75 \% \mathrm{ZnSO}_{4}+1 \% \mathrm{H}_{3} \mathrm{BO}_{3}$ & 46.33 & 129.68 & 117.18 & 48.67 \\
\hline$\left(\mathrm{T}_{13}\right) 1 \% \mathrm{ZnSO}_{4}+0 \% \mathrm{H}_{3} \mathrm{BO}_{3}$ & 46.24 & 160.16 & 144.43 & 49.77 \\
\hline$\left(\mathrm{T}_{14}\right) 1 \% \mathrm{ZnSO}_{4}+0.5 \% \mathrm{H}_{3} \mathrm{BO}_{3}$ & 46.12 & 158.44 & 142.91 & 51.87 \\
\hline$\left(\mathrm{T}_{15}\right) 1 \% \mathrm{ZnSO}_{4}+0.75 \% \mathrm{H}_{3} \mathrm{BO}_{3}$ & 45.23 & 155.32 & 144.36 & 52.04 \\
\hline$\left(\mathrm{T}_{16}\right) 1 \% \mathrm{ZnSO}_{4}+1 \% \mathrm{H}_{3} \mathrm{BO}_{3}$ & 45.48 & 161.95 & 143.24 & 52.36 \\
\hline Sem \pm & $\mathbf{0 . 6 3 6}$ & $\mathbf{1 . 7 6 0}$ & $\mathbf{4 . 3 9 7}$ & $\mathbf{2 . 5 3 5}$ \\
\hline $\mathbf{C D ~ a t ~ 5 \%}$ & $\mathbf{N S}$ & $\mathbf{5 . 0 8 2}$ & $\mathbf{1 2 . 6 9 9}$ & $\mathbf{N S}$ \\
\hline
\end{tabular}

Figures in parentheses indicate transformed values (Arc sin)

Table.6 Effect of zinc sulphate and boric acid on boron content (ppm) of leaves During first year at various stages of the experiment

\begin{tabular}{|l|c|c|c|c|}
\hline \multirow{2}{*}{ Treatments } & \multicolumn{3}{|c|}{ Boron content (ppm) during first year } \\
\cline { 2 - 5 } & Before spray & After spray & $\begin{array}{c}\text { After rainy } \\
\text { season crop }\end{array}$ & $\begin{array}{c}\text { After winter } \\
\text { season crop }\end{array}$ \\
\hline $\begin{array}{l}\left(\mathrm{T}_{1}\right) 0 \% \mathrm{ZnSO}_{4}+0 \% \mathrm{H}_{3} \mathrm{BO}_{3} \\
(\mathrm{Control})\end{array}$ & 54.09 & 53.14 & 52.74 & 55.09 \\
\hline$\left(\mathrm{T}_{2}\right) 0 \% \mathrm{ZnSO}_{4}+0.5 \% \mathrm{H}_{3} \mathrm{BO}_{3}$ & 54.52 & 90.44 & 81.01 & 52.74 \\
\hline$\left(\mathrm{T}_{3}\right) 0 \% \mathrm{ZnSO}_{4}+0.75 \% \mathrm{H}_{3} \mathrm{BO}_{3}$ & 52.43 & 100.74 & 94.24 & 53.99 \\
\hline$\left(\mathrm{T}_{4}\right) 0 \% \mathrm{ZnSO}_{4}+1 \% \mathrm{H}_{3} \mathrm{BO}_{3}$ & 51.93 & 116.00 & 100.31 & 53.99 \\
\hline$\left(\mathrm{T}_{5}\right) 0.5 \% \mathrm{ZnSO}_{4}+0 \% \mathrm{H}_{3} \mathrm{BO}_{3}$ & 52.98 & 53.74 & 51.09 & 50.82 \\
\hline$\left(\mathrm{T}_{6}\right) 0.5 \% \mathrm{ZnSO}_{4}+0.5 \% \mathrm{H}_{3} \mathrm{BO}_{3}$ & 51.50 & 91.27 & 80.74 & 54.10 \\
\hline$\left(\mathrm{T}_{7}\right) 0.5 \% \mathrm{ZnSO}_{4}+0.75 \% \mathrm{H}_{3} \mathrm{BO}_{3}$ & 54.01 & 100.50 & 95.93 & 55.37 \\
\hline$\left(\mathrm{T}_{8}\right) 0.5 \% \mathrm{ZnSO}_{4}+1 \% \mathrm{H}_{3} \mathrm{BO}_{3}$ & 53.74 & 115.68 & 105.12 & 56.14 \\
\hline$\left(\mathrm{T}_{9}\right) 0.75 \% \mathrm{ZnSO}_{4}+0 \% \mathrm{H}_{3} \mathrm{BO}_{3}$ & 52.44 & 51.74 & 51.53 & 50.99 \\
\hline$\left(\mathrm{T}_{10}\right) 0.75 \% \mathrm{ZnSO}_{4}+0.5 \% \mathrm{H}_{3} \mathrm{BO}_{3}$ & 54.53 & 90.43 & 82.14 & 55.93 \\
\hline$\left(\mathrm{T}_{11}\right) 0.75 \% \mathrm{ZnSO}_{4}+0.75 \% \mathrm{H}_{3} \mathrm{BO}$ & & 53.14 \\
\hline$\left(\mathrm{T}_{12}\right) 0.75 \% \mathrm{ZnSO}_{4}+1 \% \mathrm{H}_{3} \mathrm{BO}_{3}$ & 53.17 & 100.19 & 95.44 & 54.79 \\
\hline$\left(\mathrm{T}_{13}\right) 1 \% \mathrm{ZnSO}_{4}+0 \% \mathrm{H}_{3} \mathrm{BO}_{3}$ & 53.14 & 116.85 & 104.37 & 54.53 \\
\hline$\left(\mathrm{T}_{14}\right) 1 \% \mathrm{ZnSO}_{4}+0.5 \% \mathrm{H}_{3} \mathrm{BO}_{3}$ & 53.01 & 53.52 & 51.01 & 50.24 \\
\hline$\left(\mathrm{T}_{15}\right) 1 \% \mathrm{ZnSO}_{4}+0.75 \% \mathrm{H}_{3} \mathrm{BO}_{3}$ & 52.53 & 92.60 & 82.53 & 54.31 \\
\hline$\left(\mathrm{T}_{16}\right) 1 \% \mathrm{ZnSO}_{4}+1 \% \mathrm{H}_{3} \mathrm{BO}_{3}$ & 52.24 & 101.19 & 96.50 & 57.01 \\
\hline Sem \pm & $\mathbf{2 . 0 1 2}$ & $\mathbf{2 . 1 7 2}$ & $\mathbf{1 . 8 4 7}$ & $\mathbf{1 . 5 0 2}$ \\
\hline $\mathbf{C D ~ a t ~ 5 \%}$ & $\mathbf{N S}$ & $\mathbf{6 . 2 7 4}$ & $\mathbf{5 . 3 3 4}$ & $\mathbf{N S}$ \\
\hline
\end{tabular}

Figures in parentheses indicate transformed values (Arc sin) 
Table.7 Effect of zinc sulphate and boric acid on boron content (ppm) of leaves During second year at various stages of the experiment

\begin{tabular}{|l|c|c|c|c|}
\hline \multirow{2}{*}{ Treatments } & \multicolumn{3}{|c|}{ Boron content (ppm) during second year } \\
\cline { 2 - 5 } & Before spray & After spray & $\begin{array}{c}\text { After rainy } \\
\text { season crop }\end{array}$ & $\begin{array}{c}\text { After winter } \\
\text { season crop }\end{array}$ \\
\hline $\begin{array}{l}\left(\mathrm{T}_{1}\right) 0 \% \mathrm{ZnSO}_{4}+0 \% \mathrm{H}_{3} \mathrm{BO}_{3} \\
(\mathrm{Control})\end{array}$ & 51.44 & 51.49 & 50.01 & 50.84 \\
\hline$\left(\mathrm{T}_{2}\right) 0 \% \mathrm{ZnSO}_{4}+0.5 \% \mathrm{H}_{3} \mathrm{BO}_{3}$ & 54.11 & 94.34 & 91.01 & 65.67 \\
\hline$\left(\mathrm{T}_{3}\right) 0 \% \mathrm{ZnSO}_{4}+0.75 \% \mathrm{H}_{3} \mathrm{BO}_{3}$ & 52.95 & 102.40 & 100.24 & 66.26 \\
\hline$\left(\mathrm{T}_{4}\right) 0 \% \mathrm{ZnSO}_{4}+1 \% \mathrm{H}_{3} \mathrm{BO}_{3}$ & 54.10 & 115.74 & 112.31 & 64.59 \\
\hline$\left(\mathrm{T}_{5}\right) 0.5 \% \mathrm{ZnSO}_{4}+0 \% \mathrm{H}_{3} \mathrm{BO}_{3}$ & 51.27 & 53.52 & 50.09 & 50.58 \\
\hline$\left(\mathrm{T}_{6}\right) 0.5 \% \mathrm{ZnSO}_{4}+0.5 \% \mathrm{H}_{3} \mathrm{BO}_{3}$ & 51.77 & 94.60 & 90.74 & 65.53 \\
\hline$\left(\mathrm{T}_{7}\right) 0.5 \% \mathrm{ZnSO}_{4}+0.75 \% \mathrm{H}_{3} \mathrm{BO}_{3}$ & 54.58 & 103.43 & 101.93 & 63.94 \\
\hline$\left(\mathrm{T}_{8}\right) 0.5 \% \mathrm{ZnSO}_{4}+1 \% \mathrm{H}_{3} \mathrm{BO}_{3}$ & 54.50 & 116.03 & 115.12 & 67.95 \\
\hline$\left(\mathrm{T}_{9}\right) 0.75 \% \mathrm{ZnSO}_{4}+0 \% \mathrm{H}_{3} \mathrm{BO}_{3}$ & 52.42 & 52.04 & 50.53 & 50.16 \\
\hline$\left(\mathrm{T}_{10}\right) 0.75 \% \mathrm{ZnSO}_{4}+0.5 \% \mathrm{H}_{3} \mathrm{BO}_{3}$ & 53.39 & 94.45 & 92.14 & 65.06 \\
\hline$\left(\mathrm{T}_{11}\right) 0.75 \% \mathrm{ZnSO}_{4}+0.75 \% \mathrm{H}_{3} \mathrm{BO}_{3}$ & 51.93 & 102.41 & 101.44 & 67.79 \\
\hline$\left(\mathrm{T}_{12}\right) 0.75 \% \mathrm{ZnSO}_{4}+1 \% \mathrm{H}_{3} \mathrm{BO}_{3}$ & 53.48 & 116.85 & 116.37 & 76.74 \\
\hline$\left(\mathrm{T}_{13}\right) 1 \% \mathrm{ZnSO}_{4}+0 \% \mathrm{H}_{3} \mathrm{BO}_{3}$ & 50.91 & 53.08 & 50.24 & 50.64 \\
\hline$\left(\mathrm{T}_{14}\right) 1 \% \mathrm{ZnSO}_{4}+0.5 \% \mathrm{H}_{3} \mathrm{BO}_{3}$ & 54.88 & 95.19 & 92.53 & 66.79 \\
\hline$\left(\mathrm{T}_{15}\right) 1 \% \mathrm{ZnSO}_{4}+0.75 \% \mathrm{H}_{3} \mathrm{BO}_{3}$ & 51.57 & 104.03 & 102.50 & 61.87 \\
\hline$\left(\mathrm{T}_{16}\right) 1 \% \mathrm{ZnSO}_{4}+1 \% \mathrm{H}_{3} \mathrm{BO}_{3}$ & 52.05 & 118.35 & 117.32 & 82.26 \\
\hline Sem \pm & $\mathbf{1 . 4 6 6}$ & $\mathbf{7 . 1 1 5}$ & $\mathbf{1 . 8 4 8}$ & $\mathbf{7 . 5 4 7}$ \\
\hline $\mathbf{C D ~ a t ~ 5 \%}$ & $\mathbf{N S}$ & $\mathbf{2 0 . 5 5}$ & $\mathbf{5 . 3 3 6}$ & $\mathbf{N S}$ \\
\hline
\end{tabular}

Figures in parentheses indicate transformed values (Arc sin)

Manchanda (1974) also recorded zinc content in leaves four to seven times more than the control. Foliar application of $\mathrm{ZnSO}_{4}$ on litchi considerably increased the zinc content of the leaves (Nijjar et al., 1976). Lal et al., (2000) reported that the foliar spray of $\mathrm{ZnSO}_{4}$ at $4 \mathrm{~g}$ per plant per year significantly increased $\mathrm{Zn}$ content of leaves in guava cv. Allahabad Safeda. Perveen and Hafeez-ur-Rehman (2000) also reported that foliar application of zinc significantly increased leaf zinc contents and fruit yield as compared to trees where zinc was not included in foliar spray. Khorsandi et al., (2009) also reported increase in the $\mathrm{Zn}$ concentration of pomegranate leaves. Yadav et al., (2010) recorded maximum $\mathrm{Zn}$ content in leaf with RDF $(200+90+200$ NPK g/plant $)+40$ g Zn EDTA $+20 \mathrm{~g} \mathrm{MnSO} 4+5 \mathrm{~g} \mathrm{CuSO} 4+10 \mathrm{~g}$ Borax/plant. Khan et al., (2012) also reported that combine application of boric acid $(0.3 \%)$ and zinc sulphate $(0.5 \%)$ at fruit set stage effectively improved the B and $\mathrm{Zn}$ level in the leaves of Feutrell's early madarin. Rajkumar et al., (2014) reported that zinc sulphate and boric acid showed beneficial effect on fruit set and reducing fruit drop might be due the higher availability of photosynthates and synthesis of auxins hormones necessary for fruit set and fruit growth.

\section{Boron (B)}

The data presented in Tables 6 and 7 clearly indicated that boron content increased significantly with the increase in boron concentration during both the years of investigation but the interaction was nonsignificant. Maximum boron content (116.85 ppm) was observed in treatment $T_{12}$ which was at par with $\mathrm{T}_{4}, \mathrm{~T}_{8}$ and $\mathrm{T}_{16}$, while minimum (51.74 ppm) was observed under $\mathrm{T}_{9}$ after foliar spray. All the treatments except $\mathrm{T}_{1}$, $\mathrm{T}_{9}$ and $\mathrm{T}_{13}$ were found significant over treatment $\mathrm{T}_{9}$. After harvesting of rainy season crop, boron content of leaves differed 
significantly with various levels of boron concentration. The maximum boron content (107.32 ppm) was observed in treatment $\mathrm{T}_{16}$, while, minimum (51.01 ppm) was found in treatment $\mathrm{T}_{5}$. When leaf boron content was observed after winter season crop it differed non-significantly with foliar application of boron and zinc. Similar results were recorded during $2^{\text {nd }}$ year of study. These findings are in agreement with the earlier findings of Maksoud and Haggag (1996) who did not find significant effect of foliar spray of boron on leaf boron content in apples.

Although, Shukla (1983) reported a synergistic relationship between zinc and boron content and observed an increment in the zinc content followed by an increase in boron concentration. Delgado et al., (1994) suggested that B was mobilized from young leaves during anthesis to supply the needs of flowers and fruits in olive trees.

Perveen and Hafeez-ur-Rehman (2000) found that application of boron significantly increased total yield, but did not influence leaf boron content. While, foliar sprays of $\mathrm{Mn}, \mathrm{Zn}$ and $\mathrm{B}$ with urea not affected the B concentration in sweet orange leaves (Tariq et al., 2007).

The results indicated that the doses of zinc sulphate $(1.0 \%)$ and boric acid $(1.0 \%)$ individually or in combination in the month of July were found most effective to enhance the vegetative growth, flowering, fruiting, fruit yield and fruit quality of guava cv. Pant Prabhat. The leaf nutrient status of guava leaves was also influenced by the external application of zinc sulphate and boric acid.

\section{Acknowledgements}

The authors are thankful to the Head, Department of Horticulture, for providing the required research facilities.

\section{References}

Bhargava, B.S. and Chadha, K.L. 1993. Leaf nutrient guide for fruit crops. In: Advances in Horticulture, $2^{\text {nd }}$ Vol. (Chadha, K.L. and Pareek, O.P., eds). Malhotra Publishing House, New Delhi, PP. 973-1029.

Chhonkar, V.S. and Singh, P.N. 1981. Effect of nitrogen, phosphorus and potash as foliar spray on growth, flowering and fruiting of guava (Psidium guajava L.). Punjab horticultural journal, 23 (1/2): 34-37.

Das, D.K. 2003. Micronutrients: Their behaviors in soils and plants. Kalyani publication, Ludhiana pp. 1-2.

Delgado, A., Benlloch, M. and FernandezEscobar, R. 1994. Mobilization of boron in olive trees during flowering and fruit development. Horticultural Science, 29: 616-618.

Embleton, T.W., Wallihan, E.F. and Goodall, G.F. 1965. Effectiveness of soil vs. foliar applied zinc and of foliar appied manganese on Calfornaia lemons. Proceedings of the American Society for Horticultural Science, 86: 253-59.

Hassan, H.S.A., Sarrwy, S.M.A. and Mostafa, E.A.M. 2010. Effect of foliar spraying with liquid organic fertilizer, some micronutrients and gibberellins on leaf minerals content, fruit set, yield, and fruit quality of 'Hollywood' plum trees. Agriculture and Biology Journal of North America, 1: 638-643.

Jackson, M.L. 1973. Soil chemical analysis. Asia Publishing House, New Delhi.

Kanwar, J.S. and Dhingra, D.R. 1962. Effect of micronutrient sprays on the chemical composition of citrus leaves and incidence of chlorosis. Indian Journal of Agricultural Sciences, 32: 309-14.

Khan, A.S., Ullah, W., Malik, A.U., Ahmad, R., Saleem, B.A. and Rajwana, I.A. 2012. Exogenous applications of boron 
and zinc influence leaf nutrient status, tree growth and fruit quality of Feutrell's early (Citrus reticulata blanco). Pakistan Journal of Agricultural Sciences, 49(2), 113119.

Khorsandi, F., AlaeiYazdi, F. and Vazifehshenas, M.R. 2009. Foliar zinc fertilization improves marketable fruit yield and quality attributes of pomegranate. International Journal of Agriculture and Biology, 11 (6):766770 .

Lal, G., Sen, N.L. and Jat, R.G. 2000. Yield and leaf nutrient composition of guava as influenced by nutrients. Indian Journal of Horticulture. 57(2):130-132.

Lindsay, W.L. and Norvell, W.A. 1978. Development of DTPA soil test for zinc, iron, manganese and copper. Soil Science Society of America Journal, 42: 421-28.

Maksoud, M.A. and Haggag, I.F. 1996. Effect of boron sprays on yield of apples. Egyptian Journal of Horticulture, 23(2): 191-195.

Manchanda, H.R. 1974. Chemical composition of sweet orange leaves (Citrus sinensis L. asbeck) as affected by foliar application variety Pineapple. Indian Journal of Horticulture, 31 (1): 34-37.

Manchanda, H.R., Randhawa, N.S. and Shukla, U.C. 1971. Effect of foliar application of different micro-nutrients in relation to sources of nitrogen on chemical composition of sweet orange levels (Citrus sinesis Osbeck) var. Blood Red. Indian Journal of Horticulture, 28: 100-107.

Nijjar, G.S. and Brar, S.S. 1977. Comparison of soil and foliar applied zinc in Kinnow (mandarin). Indian Journal of Horticulture, 34 (2):130-36.

Nijjar, G.S., Arora, J.S., Singh, G. and Dwivedi, RS. 1976. Symptoms of zinc deficiency in Mango. The Punjab horticultural journal, 16 (3\&4):113-114. Perveen, S. and Hafeez-ur-Rehman. 2000. Effect of foliar application of zinc, manganese and boron in combination with urea on the yield of sweet orange. Pakistan Journal of Agricultural Research, 16(2): 135-141.

Prakash, J., Singh, N.P. and Sankaran, M. 2006. Influence of nitrogen, $\mathrm{Zn}$ and $\mathrm{B}$ on leaf nutrient composition and yield of guava cv. L-49 (Psidium guajava L.). Environment and Ecology, 24S (4): 1158-1160.

Rajkumar; Tiwari, J.P. and Lal, Shant. 2014. Effect of zinc and boron on fruit yield and quality of winter season guava (Psidium guajava L.) cv. Pant Prabhat. Annals of Agri-Bio Research, 19(1): 105-108.

Rajkumar, Kaur, G., Mann, A., Lata, C., Singh, A and Kumar, A. 2016. Biochemical changes in guava (Psidium guajava L.) cv. Allahabad Safeda fruits at different maturity stages during summer and winter seasons. Indian Journal of Agricultural Sciences, 86 (12): 1595-1600

Sharma, A.S., Sehrawat, S.K., Singhrot, R.S. and Boora, K.S. 2007. Assesment of genetic diversity and relationship among Psidium spp. through RAPD analysis. Acta Horticulturae, 735: 7178.

Sharma, R. and Bhattacharya, R.K. 1989. Effect of foliar nutrition of zinc on the nutrient concentration of guava leaves. South Indian horticulture, 37 (6): 323 325.

Shukla, M.P. 1983. Sulphur, zinc and boron nutrition of Rai (Brassica juncea). Journal of the Indian Society of Soil Science, 31: 517-520.

Singh, R.R., Joon, M.S. and Daulta, B.S. 1983. A note on the effects of foliar spray of urea and boric acid on physico- 
chemical composition of guava fruits cv. Lucknow-49. Haryana Journal of Horticultural Sciences, 12 (1-2): 68-70.

Smith, P.F. 1967. Leaf analysis of citrus. In: Fruit nutrition Chap. 8, N.F. Childers Ed. Somerset Press, Somerville, New Jersey, pp. 207-228.

Srivastava, A.K. and Singh, S. 2005. Boron nutrition in citrus-current status and future strategies - review. Agricultural Reviews, 26(3): 173-186.

Supriya, L. and Bhattacharya, R.K. 1995. NPK contents of Assam lemon leaf as affected by foliar zinc spray. Annals of
Agricultural Research, 16 (4): 193-94. Tariq, M., Sharif, M., Shah, Z. and Khan, R. 2007. Effect of Foliar Application of Micronutrients on the Yield and Quality of Sweet Orange (Citrus sinensis L.). Pakistan Journal of Biological Sciences, 10: 1823-1828. Yadav, M.K., Patel, N.L., Parmar, B.R., Kirtibardhan and Paramver, S. 2010. Effect of micronutrients on growth and crop duration of banana cv. Grand Nain. Progressive Horticulture, 42(2):162165.

\section{How to cite this article:}

Rajkumar, J.P. Tiwari, Shant Lal, Mohit Kumar, Anshuman Singh and Ashwani Kumar. 2017. Effect of Boron and Zinc Application on Nutrient Uptake in Guava (Psidium guajava L.) cv. Pant Prabhat Leaves. Int.J.Curr.Microbiol.App.Sci. 6(6): 1991-2002. doi: https://doi.org/10.20546/ijcmas.2017.606.234 